\title{
Transcranial ultrasonic wave propagation simulation: skull insertion loss and recovery
}

Lanbo Liu, Kuang He, Lihong V. Wang

Lanbo Liu, Kuang He, Lihong V. Wang, "Transcranial ultrasonic wave propagation simulation: skull insertion loss and recovery," Proc. SPIE 6437, Photons Plus Ultrasound: Imaging and Sensing 2007: The Eighth Conference on Biomedical Thermoacoustics, Optoacoustics, and Acousto-optics, 64370X (13 February 2007); doi: 10.1117/12.698237

SPIE. Event: SPIE BiOS, 2007, San Jose, California, United States 


\title{
Transcranial ultrasonic wave propagation simulation: skull insertion loss and recovery
}

\author{
Lanbo Liu* ${ }^{\mathrm{a}}$, Kuang He ${ }^{\mathrm{a}}$, Lihong V. Wang ${ }^{\mathrm{b}}$ \\ ${ }^{a}$ School of Engineering, University of Connecticut, 261 Glenbrook Rd., Storrs, CT, USA 06269; \\ ${ }^{b}$ Dept. of Biomedical Engineering, Washington University, 9876 Light Ave., St. Louis, MO USA \\ 00555-9642
}

\begin{abstract}
Severe energy dissipation and waveform aberration in ultrasonic wave propagation due to the human skull remain major challenges to achieving good focus in high intensity focused ultrasound (HIFU) brain therapy and high-resolution thermoacoustic tomography (TAT) of the human brain. With the inclusion of the skull insertion loss, we numerically simulated the ultrasonic wave propagation using the pseudospectral time domain (PSTD) algorithm for TAT setup. We then applied the redatuming scheme through downward and upward continuation originated in seismic signal processing to eliminate the diffraction caused by the irregularity of the skull and to recover the insertion loss due mainly to the diploes layer of the skull. This approach, after further validation, is aimed to recover wave energy dissipation and waveform aberration in ultrasonic measurements applied to both trans-skull imaging (ultrasound propagates outward) and therapy (ultrasound propagates inward).
\end{abstract}

Keywords: ultrasonic wave, insertion loss, skull, numerical simulation, redatuming, downward and upward continuation

\section{INTRODUCTION}

Ultrasound applications in biomedical engineering fall into two principal areas: diagnostic imaging [1] and therapy [2]; the two differ in the power, intensity, and duration of the ultrasound [3]. However, the fundamental physics of the ultrasonic wave propagation stays the same. Ultrasound diagnosis and therapeutic treatment have made tremendous progress in the last quarter century, mainly due to the explosive advances in microelectronics and computer science and engineering. Nevertheless, the success stories are mainly associated with the diagnosis and treatment of organs and body parts that do not have heavy bone coverage, such as the kidney, liver, prostate, etc. [3]. When we attempt to use ultrasound to image or treat disorders, tumors or other diseases in the human brain, the shielding effect caused by the high contrast between the human skull and brain tissue remains the biggest obstacle from the infancy of this technique [4]. An earlier solution for overcoming ultrasound transmission blockage by the skull was to create an acoustic "window" by removing a small portion of skull bone. Obviously, this practice caused additional discomfort and imposed more danger for the patient. An effective noninvasive approach is urgently needed for both bringing ultrasound energy into the brain for therapy and surgery, and retrieving ultrasound energy from inside the skull for diagnostic imaging.

Forward modeling with analytic or semi-analytic approaches was used to study ultrasonic wave propagation through the human skull as a multi-layered medium [5]. Recently, fully numerical simulation using a finite difference time domain (FDTD) method was also introduced to model trans-skull ultrasonic wave propagation [6]. In this paper, we first employed the pseudospectral time domain (PSTD) method $[7,8]$ to generate trans-skull ultrasonic wave numerically in the context of thermoacoustic tomography (TAT, $[9,10])$. Theoretically, PSTD is more accurate and more efficient than FDTD. The physical ultrasonic source generated by the microwave heating impulse in TAT was mimicked by the instantaneous firing of thousands of individual ultrasonic impulses distributed over the entire numerical grids occupied by human brain. Second, the PSTD forward-modeling generated time domain series of the ultrasonic waves recorded at the transducer array outside the skull were performed redatuming through downward and upward continuation [11, 12] to reduce the effect of the scattering of the irregular shape of the skull and the insertion loss of the diploes layer. The redatuming algorithm provides a viable way to correct wave distortion for obtaining high resolution in TAT imaging.

*lanbo.liu@uconn.edu; phone 1860 486-1388; fax 1860 486-2298; engr.uconn.edu/ lanbo

Photons Plus Ultrasound: Imaging and Sensing 2007: The Eighth Conference on Biomedical Thermoacoustics, Optoacoustics, and Acousto-optics, edited by Alexander A. Oraevsky, Lihong V. Wang,

Proc. of SPIE Vol. 6437, 64370X, (2007) · 1605-7422/07/\$18 · doi: 10.1117/12.698237

Proc. of SPIE Vol. 6437 64370X-1 


\section{PSTD NUMERICAL SIMULATION}

\subsection{Human brain model}

A generic human brain model (Figure 1) was digitized to generate a 2-dimensional (2D) numerical model for being used in the PSTD ultrasonic wave propagation simulation. The model is a simplified 2D slice sub-paralleling to the Occiput plane as shown in Figure 1b. The use of a 2D model can be justified by arguing that the structure of the skull-brain system has few changes in the direction perpendicular to the model plane within a couple of wavelengths of the ultrasound (less than a centimeter). A three-layer skull model was used to simulate the outer table (outer ivory), the diploes (the marrow), and the inner table (inner ivory). Since our purpose here is to study the influence of the skull on the propagation of ultrasonic waves, we assumed a uniform brain inside the skull to simplify the treatment, but the ultrasonic source energy level is proportional to the firmness (or the brightness in the MRI image) of brain tissues. Nevertheless, the PSTD algorithm can readily handle any arbitrarily distributed acoustic property specifications of a skull-brain system.

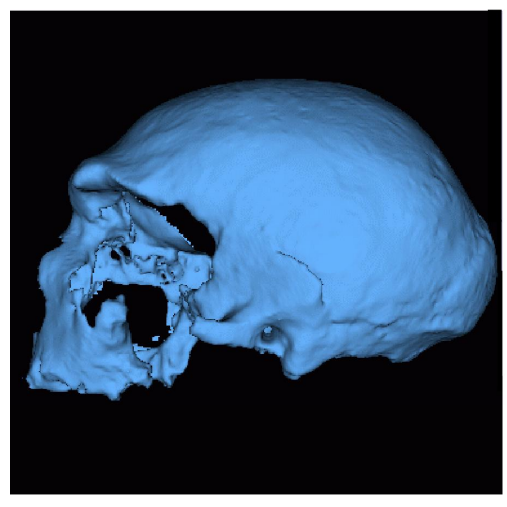

(a)

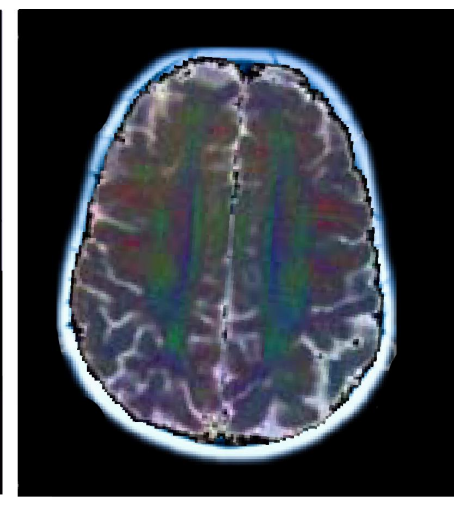

(b)

Fig. 1. A generic 3D skull (a) and the 2D skull-brain model in axial imaging plane (b) used in the PSTD simulation based on an MRI imaging slice.

It is a common practice in both imaging and therapy to place the sample in water or another fluid to increase the coupling efficiency between the medium and the ultrasonic transducers through minimizing the contrast in acoustic impedance between the medium and the skull. We assumed the medium to be water in our PSTD simulation. The acoustic parameters of the water-skull-brain system were gathered together from published sources in the literature [3-6], as listed in Table 1. The value of the skull insertion loss is based on the measured data for ultrasonic wave with a central frequency of $0.25 \mathrm{MHz}[4]$.

Table 1. Acoustic parameters of the water-skull-brain system model.

\begin{tabular}{|l|l|l|l|l|l|}
\hline Skull Model & Water & Outer Table & Diploes & Inner Table & Brain \\
\hline Thickness $(\mathrm{mm})$ & & 2.0 & 2.0 & 2.0 & \\
\hline Density $\left(\mathrm{kg} / \mathrm{m}^{3}\right)$ & 998 & 1910 & 1740 & 1870 & 1030 \\
\hline Sound Speed $(\mathrm{m} / \mathrm{s})$ & 1500 & 2900 & 2500 & 2900 & 1545 \\
\hline Impedance $\left(\mathrm{kg} / \mathrm{m}^{3} \mathrm{~s}\right)$ & $1.5 \times 10^{6}$ & $5.54 \times 10^{6}$ & $4.35 \times 10^{6}$ & $5.42 \times 10^{6}$ & $1.61 \times 10^{6}$ \\
\hline Insertion Loss $(\mathrm{Np} / \mathrm{m})$ & 0.0 & 2.0 & 22.5 & 2.0 & 1.0 \\
\hline
\end{tabular}

The 2D PSTD algorithm for the TAT transducer-skull-brain model has a total of $256 \times 256$ grids. The grid size is $\mathrm{dx}=$ dy $=1.0 \mathrm{~mm}$. A duration of $100 \mu \mathrm{s}$ (microseconds) was used to record the synthetic waveform at the receiving points with a time step of $0.02 \mu \mathrm{s}$. A source impulse function, known as the Ricker wavelet [13], with a central frequency of $0.25 \mathrm{MHz}$ was fired simultaneously at all grids inside the skull with the ultrasonic level proportional to brain tissue firmness. A group of 240 transducer locations forms a complete circular array, with a radius of $117 \mathrm{~mm}$, to record the time domain ultrasonic pressure. For the ultrasonic impulse with a central frequency of $0.25 \mathrm{MHz}$, the dominant wavelength is $6 \mathrm{~mm}(\lambda=\mathrm{vT}=\mathrm{v} / \mathrm{f}=1500(\mu \mathrm{m} / \mu \mathrm{s}) /(0.25 / \mu \mathrm{s})=6.0 \mathrm{~mm})$. Thus, for an arc length of $3 \mathrm{~mm}$ between 2 adjacent 
transducers on the circle $(\Delta \mathrm{l}=\mathrm{r} \Delta \theta=117 \times 2 \pi / 360=3.06 \mathrm{~mm})$, the spatial sampling rate is adequate to assure two receiving points per wavelength for reconstructing brain images without numerical aliasing.

\subsection{PSTD numerical simulation results}

Figure 2 shows the simulated waveforms recorded at the 240 receivers on the circular receiver array for cases without (Figure 2a) and with (Figure 2b) diploes insertion loss. The amplitude of the ultrasound level for the case of with insertion loss (Figure 2b) has been exaggerated 100 times to be comparable with the case of without insertion (Figure 2a). More importantly, for the insertion loss preserved case, the continuity of waveform features among the receivers has been severely degraded due to the insertion loss of skull diploes. Certain degree of focusing-defocusing of ultrasonic energy can also be easily observed.
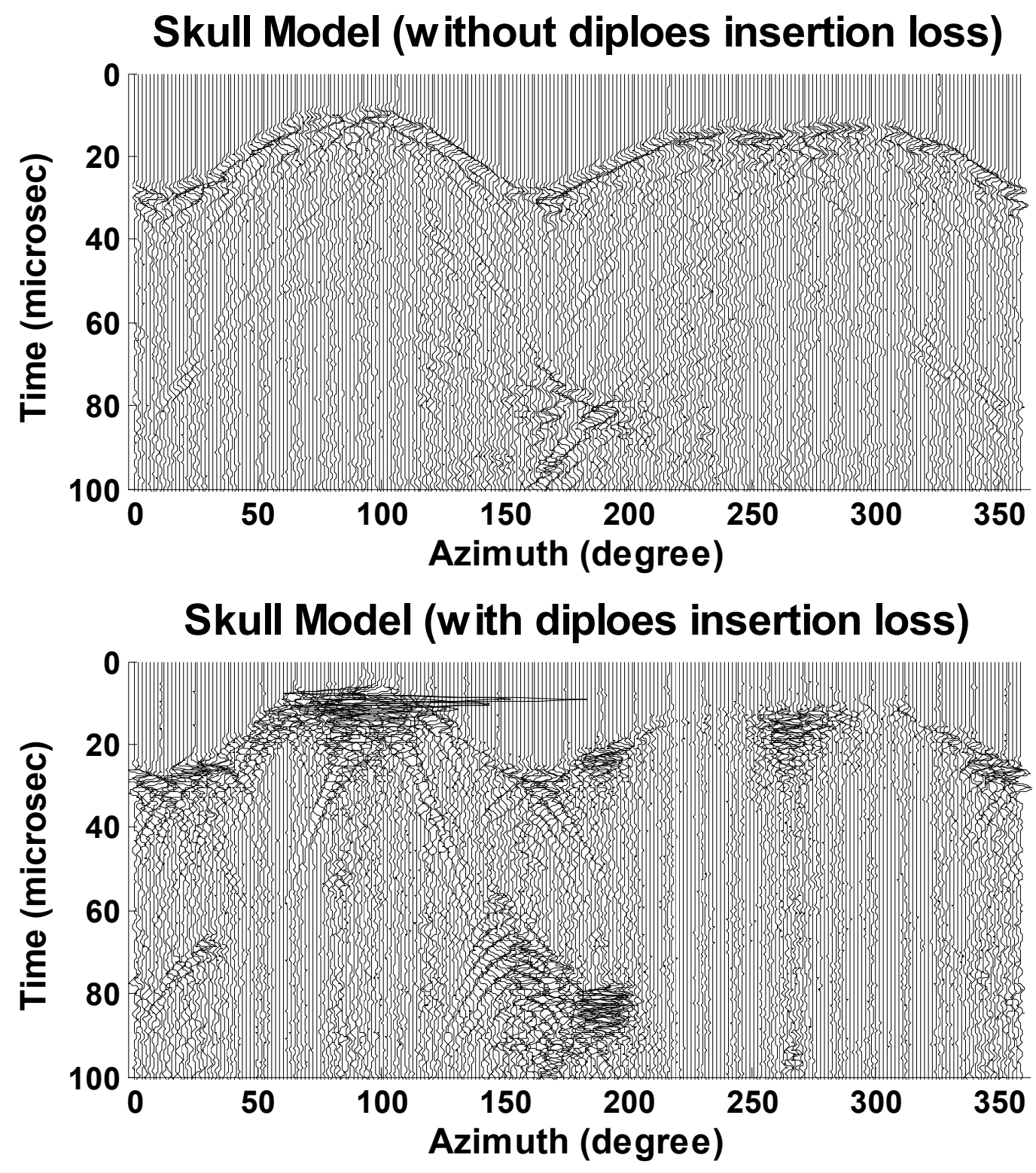

Fig. 2. PSTD numerical simulation results of ultrasonic time series recorded at the circular transducer array with 240 recording positions. The recording setup is shown in Figure 1b. 


\section{DIFFRACTION ELIMINATION AND INSERTION LOSS RECOVERY BY REDATUMING THROUGH DOWNWARD-UPWARD CONTINUATION}

\subsection{Redatuming through downward/upward continuation}

In geophysical exploration, one effective way to eliminate the adverse effects of the heterogeneous surface layer on image reconstruction of targets in deeper formations (or later times in a seismic profile) is shifting the reference datum from the original one (the surface on which the seismic data were acquired) to an imaginary interface at a greater depth (or later time in a seismic profile) to exclude the heterogeneous surface layer. This process is called the downward continuation [14]. Once the effect of the surface layer can be modeled and separated, a more uniform layer can be virtually put back and the datum can be shifted back to the original one. This procedure is called the upward continuation, and the whole process is referred as redatuming through downward and upward continuations [14]. In this section we apply the redatuming scheme based on the full wave-equation $[11,12]$ to the synthetic ultrasonic data generated by the PSTD simulation to suppress the adverse effect in ultrasonic wave energy and waveform caused by the skull layers.

The redatuming approach uses the Kirchhoff integral formulation of the wave equation [11]. The implementation of the wave-equation redatuming is essentially a precise and efficient computerized form of the Huygens principle. Unlike redatuming with static shifts, wave-equation redatuming removes the distortions caused by material interface irregularity in a manner consistent with wavefield propagation. This ensures that subsequent processing steps that assume hyperbolic form, or even more complicated trajectories consistent with wave propagation, can be accurately applied. As an illustration, Figure 4 shows the procedure of redatuming. The material property model corresponding to the original Berryhill model [11] is shown as Figure 4a. The model contains a surface layer with an irregular bottom interface, and 2 linear reflectors are located in the sub-stratum: one horizontal and one with 16-degree dip to the right (Figure 4a). The original synthetic reflection (sources and receivers are all located at zero-depth) record is shown in Figure 4b. After the procedure of downward continuation (Figure 4c), and the complete downward-upward continuations (Figure 4d), it is clear that the adverse effect of the irregular interface on the 2 linear reflectors in deeper formation has been greatly eliminated, and the two linear reflectors were fundamentally recovered and become linear appearance again.

(a) Berryhill Model

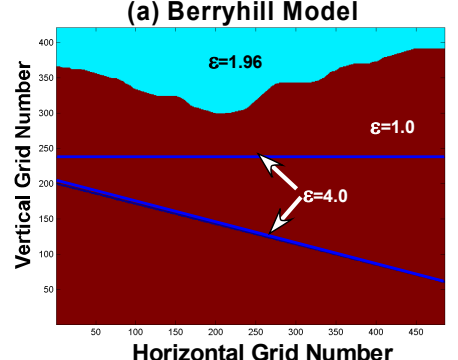

(b) Original Record

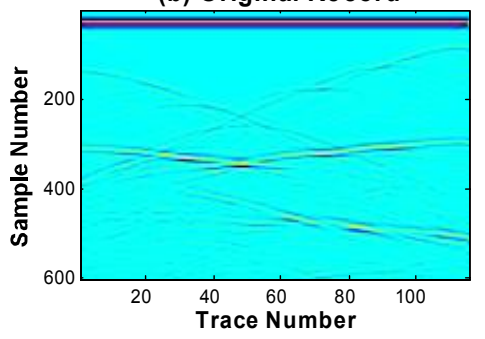

(c) w/ Downward Continu.

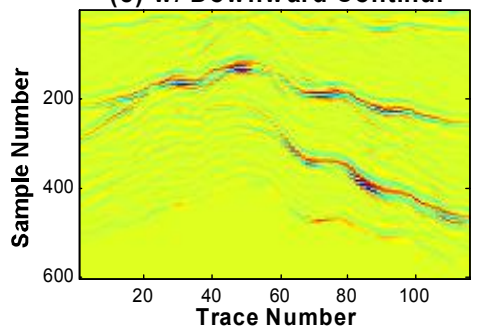

(d) w/ Down/upward Continu.

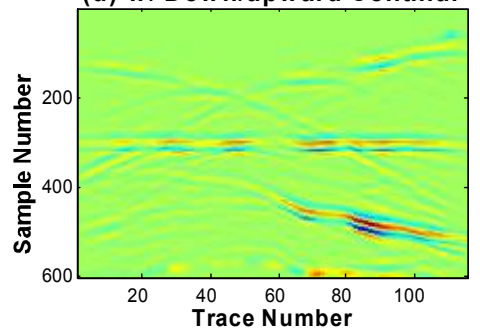

Fig. 4. The original model (a) and the results of wave-equation redatuming (b-d) for the original Berryhill model [11]. The model contains a surface layer (in light blue color) with an EM velocity 1.4 times slower than the sub-stratum (dark red). The 2 linear reflectors have a velocity half of that in the sub-stratum. After redatuming the 2 straight linear reflectors recovers two linear lines (d). 


\subsection{Redatuming with the insertion loss compensated}

In biomedical engineering applications, the ultrasonic waves propagate in either the inside-out fashion for diagnosis, or the outside-in manner for therapy; the ultrasonic waves only travel one-way. This kind of one-way propagation setup reduces the energy loss and scattering effect of the skull to one half, compared with the reflection setup as shown in Figure 4, and corresponds to the data collection fashion of pre-stacking in geophysical exploration [15]. The one-way setup is the biomedical engineers' contribution to reduce the adverse effect in ultrasonic wave propagation via hardware innovation. The redatuming scheme is the applied mathematicians' contribution via processing algorithm software improvement.

(a)

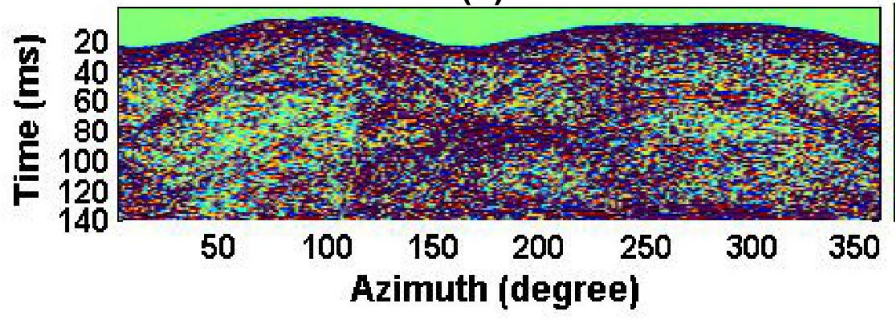

(b)

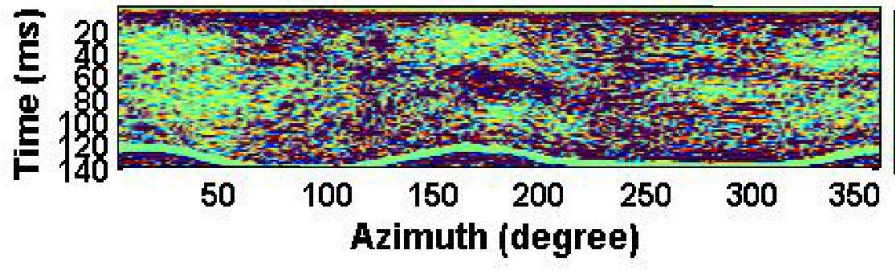

(c)

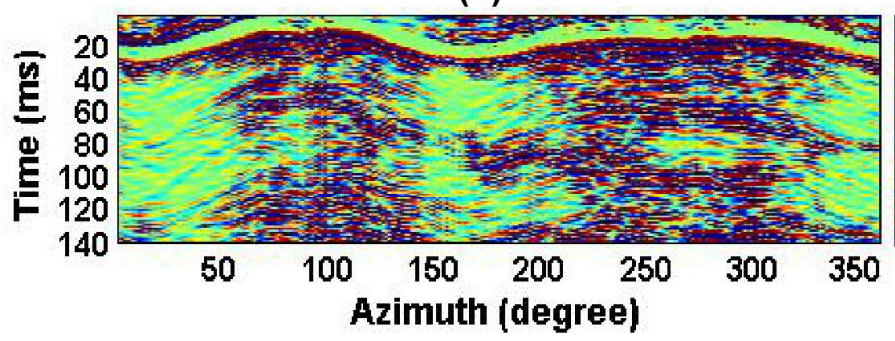

(d)

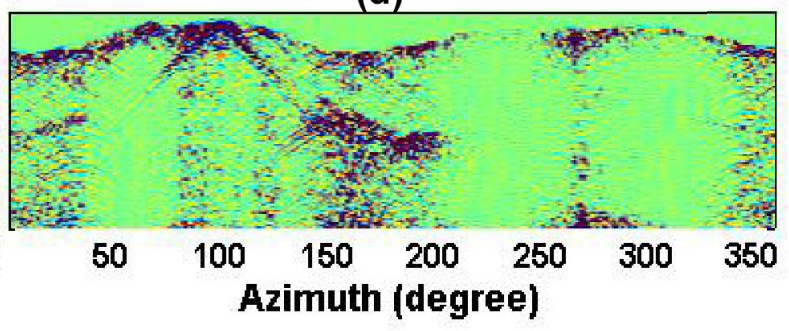

(e)

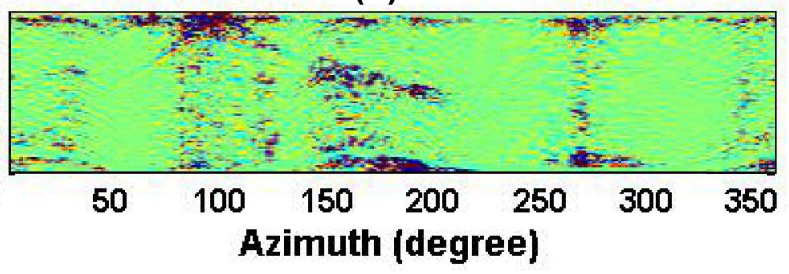

(f)

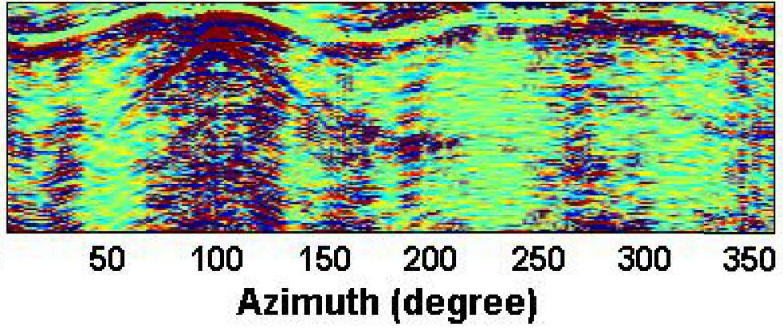

Fig. 5. Comparison of the redatuming results based on the simulated time domain records for the cases of with (a-c) and without (d-f) diploes insertion loss. The original synthetic results (a and d) were downward continued (b and e), then upward continued ( $\mathrm{c}$ and $\mathrm{f}$ ) to complete the redatuming procedure.

In Berryhill's original approach [11, 15], the media were assumed to be lossless, only wavefield distortion caused by interface irregularity was included in the redatuming process. In our approach shown in this paper, we have also loosen the lossless assumption, and added the algorithm to recover the insertion loss due to the diploes layer of the skull by assuming the loss is exponential with respect to the length of the ray path, with the loss coefficient given in Table 1. Figure 5 shows the Comparison of the redatuming results based on the PSTD simulated time series recorded at the circular array with 240 transducer positions for the cases of with and without diploes insertion loss. The redatuming process is shown in the left column for the synthetic records without insertion loss, and in the right column for the synthetic records with diploes insertion loss. Comparison of Figure $5 \mathrm{f}$ for the case of including insertion loss with Figure $5 \mathrm{c}$ for the case of excluding insertion loss, it becomes apparent that with the redatuming process considering both 
scattering and absorptive attenuation, the redatuming-processed records with inclusion of the insertion loss contains the majority of wavefield features shown in the records with the insertion loss excluded. The redatuming-processed records should be ready for further processing to carry out the tomographic image reconstruction and be expected to reveal features in human brain that interests to clinic diagnosis.

\section{RESULT DISCUSSION AND CONCLUSION}

With the aid of numerical simulation, we have applied the scheme of wave-equation redatuming through downward and upward continuation to recover ultrasonic energy dissipation and waveform distortion in TAT ultrasonic measurement due to scattering and insertion loss of human skull. The main purpose is to enhance the targets in later times of a ultrasonic data set for a more focused imaging and more confident target characterization in human brains. The example and results of wave-equation redatuming presented in this paper demonstrate that with further investigation and improvement, this method has the potential to suppress the diffractions and interferences caused by the geometric irregularity and the material property heterogeneity (insertion loss) in the outmost surface layer (human skull).

\section{REFERENCES}

1. G. T. Clement, P. J. White, R. L. King, N. McDannold, and K. Hynynen, "An MRI-compatible large scale array for trans-skull ultrasound surgery and therapy," J. Ultrasound in Medicine 24, 1117-1125 • 0278-4297 (2005).

2. G. ter Haar, "Acoustic surgery," Physics Today 29-34, (2001).

3. J. Sun, and K. Hynynen, "Focusing of therapeutic ultrasound through a human skull: A numerical study," J. Acoust. Soc. Am. 104, 17075-1715 (1998).

4. F. J. Fry, and J. E. Barger, "Acoustical properties of the human skull," J. Acoust. Soc. Am. 63(5), 1576-1590 (1978).

5. M. Hayner, and K. Hynynen, "Numerical analysis of ultrasonic transmission and absorption of oblique plane waves through the human skull," J. Acoust. Soc. Am. 110, 3319-3330 (2001).

6. M. Pernot, J. Porquet, C. Saint Olive, F. Vignon, D. Cassereau, J.-F. Aubry, M. Tanter, and M. Fink, "3D finite difference simulation of coupled acoustic wave and bio-heat equation: skull heating prediction for non-invasive brain HIFU therapy," WCU 2003, Paris, September 7-10 (2003).

7. L. Liu, and S. Arcone, "Propagation of radar pulses from a horizontal dipole in variable dielectric ground: A numerical approach," Subsurface Sensing Technologies and Applications 6, 5-24 (2005).

8. L. Liu, and L. V. Wang, "High fidelity simulation of ultrasonic wave propagation in a 2-dimensional brain model for microwave-induced thermoacoustic tomography," Proceedings of the $9^{\text {th }}$ Western Pacific Acoustics Conference Seoul, June 26-28, (2006).

9. M. Xu, G. Ku, and L. V. Wang, "Microwave-induced thermoacoustic tomography using multi-sector scanning," Med. Phys. 28, 1958-1963 (2001).

10. L. V. Wang, X. Zhao, H. Sun, and G. Ku, "Microwave-induced acoustic imaging of biological tissues," Rev. Sci. Instru. 70, 3744-3748 (1999).

11. J. R. Berryhill, "Wave-equation datuming," Geophysics 44, 1329-1344 (1979).

12. L. Liu, K. He, X. Xie, and J. Du, "Image enhancement with wave-equation redatuming: Application to GPR data collected at public transportation sites," J. Geophys. Eng. 3, 1-9 (2007).

13. R. E. Sheriff, and L. P. Geldart, Exploration Seismology, Cambridge University Press, Cambridge, UK, 1995.

14. J. Claerbout, Basic Earth Imaging, Stanford University, Stanford, California, 2001.

15. J. R. Berryhill, "Wave-equation datuming before stack," Geophysics 49, 2064-2066 (1984). 